\title{
Five-element Johann-type x-ray emission spectrometer with a single-photon-counting pixel detector
}

\author{
Evgeny Kleymenov, ${ }^{1,2, a)}$ Jeroen A. van Bokhoven, ${ }^{1,2}$ Christian David, ${ }^{1}$ Pieter Glatzel, ${ }^{3}$ \\ Markus Janousch, ${ }^{1}$ Roberto Alonso-Mori, ${ }^{4}$ Marco Studer, ${ }^{1}$ Markus Willimann, ${ }^{1}$ \\ Anna Bergamaschi, ${ }^{1}$ Beat Henrich, ${ }^{1}$ and Maarten Nachtegaal $\left.{ }^{1, b}\right)$ \\ ${ }^{1}$ Paul Scherrer Institut, 5232 Villigen, Switzerland \\ ${ }^{2}$ ETH Zurich, Institute for Chemical and Bioengineering, 8093 Zurich, Switzerland \\ ${ }^{3}$ European Synchrotron Radiation Facility, 6 Rue Jules Horowitz, 38043 Grenoble, France \\ ${ }^{4}$ SLAC National Accelerator Laboratory, 2575 Sand Hill Road, Menlo Park, California 94025, USA
}

(Received 24 March 2011; accepted 19 May 2011; published online 16 June 2011)

\begin{abstract}
A Johann-type spectrometer with five spherically bent crystals and a pixel detector was constructed for a range of hard x-ray photon-in photon-out synchrotron techniques, covering a Bragg-angle range of $60^{\circ}-88^{\circ}$. The spectrometer provides a sub emission line width energy resolution from sub-eV to a few $\mathrm{eV}$ and precise energy calibration, better than $1.5 \mathrm{eV}$ for the full range of Bragg angles. The use of a pixel detector allows fast and easy optimization of the signal-to-background ratio. A concentration detection limit below $0.4 \mathrm{wt} \%$ was reached at the $\mathrm{Cu} \mathrm{K} \alpha_{1}$ line. The spectrometer is designed as a modular mobile device for easy integration in a multi-purpose hard x-ray synchrotron beamline, such as the SuperXAS beamline at the Swiss Light Source. (C) 2011 American Institute of Physics. [doi:10.1063/1.3600452]
\end{abstract}

\section{INTRODUCTION}

Synchrotron-based techniques using hard $\mathrm{x}$-ray radiation enable the determination of the geometric and/or electronic structure of materials under operating conditions because of the large penetration depth of hard x-rays. This makes studies possible under in situ conditions, which are crucial for determining structure-property relationships of catalysts, whose chemical state and structure depend on factors, such as gas composition, temperature, and pressure. $\mathrm{X}$-ray diffraction (XRD) yields mainly the long-range crystal structure. X-ray absorption spectroscopy delivers information about the local (up to $6 \AA$ ) geometric structure and the chemical state of atoms. Detection of secondary $\mathrm{x}$-ray radiation with a sub line width bandwidth enables us to extend the range of synchrotron hard x-ray techniques to photon-in photon-out techniques, ${ }^{1}$ such as $\mathrm{x}$-ray emission spectroscopy (XES), resonant inelastic X-ray scattering (RIXS), partial fluorescence yield (PFY) XAS, and X-ray Raman spectroscopy (XRS). As the line width of the $\mathrm{X}$-ray emission lines in the hard $\mathrm{X}$-ray region ranges from one to a few dozen $\mathrm{eV},{ }^{2}$ an energy bandwidth between sub-e $\mathrm{V}$ and several $\mathrm{eV}$ is required for implementation of these techniques.

Bragg diffraction by crystals is commonly used for monochromatization of hard $\mathrm{x}$-ray radiation. Curved crystals are used to increase the efficiency of the spectrometer in collecting photons. Spectrometer designs with curved crystals were first presented in the 1930s by Johann ${ }^{3}$ and von Hamos. ${ }^{4}$ Johansson also developed a spectrometer with a bent crystal of reduced aberrations, which was achieved by a sophisticated procedure of crystal production. ${ }^{5}$ These three spectrometer designs are commonly used today to

\footnotetext{
a)Electronic mail: evgeny.kleymenov@psi.ch.

b) Electronic mail: maarten.nachtegaal@psi.ch.
}

construct curved-crystal x-ray spectrometers. With the advent of high-flux third-generation synchrotron sources, practical application of photon-in photon-out techniques became possible. Several synchrotrons offer hard x-ray beamlines equipped with high-resolution emission spectrometers. ${ }^{6-17}$

The spectrometer presented here is installed at the $\mathrm{Su}-$ perXAS beamline of the Swiss Light Source. The SuperXAS beamline is equipped with a collimating mirror, followed by a fixed-exit double crystal monochromator to ensure high energy resolution. The monochromator has two crystal pairs, $\mathrm{Si}(111)$ and $\mathrm{Si}(311)$, and covers the energy range from 4.5 to $35 \mathrm{keV}$. The spectrometer design is of Johann geometry with spherically bent crystals. Five bent crystal analyzers (hereafter referred to as "crystals") are used to increase collection efficiency. The analyzers are produced in-house. The main advantage over similar spectrometer designs ${ }^{9,12}$ is the photonenergy-thresholded pixel x-ray detector, which facilitates efficient optimization of the performance of the spectrometer and suppression of the background counts without sacrificing signal intensity.

\section{SPECTROMETER DESIGN}

Figure 1 shows a scheme of a Johann-type spectrometer. A sample $(S)$, crystal center $(C)$, and detector center $(D)$ are located on a (Rowland) circle of radius $R$. The emitted x-ray radiation of wavelength $\lambda=2 d \sin (\theta) / n$ is reflected by the crystal of radius of curvature $2 R$ and focused onto the detector. Here, $n$ is a positive integer referred to as "order of diffraction," $d$ a crystal lattice constant, and $\theta$ an incident angle relative to the crystal surface, the "Bragg angle." The exact focusing requirement is met only for the center of the crystal; therefore, this optical scheme includes aberrations. The contributions of the aberrations increase with increasing crystal 


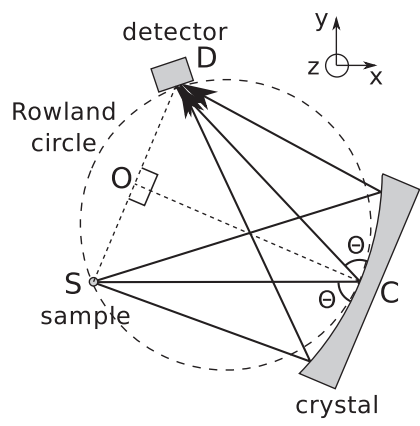

FIG. 1. Scheme of the Johann geometry of an x-ray emission spectrometer. Objects not to scale.

diameter $d$, decreasing radius of curvature $2 R$, and decreasing Bragg angle $\theta$.

Five identical crystals were used to increase the collected X-ray flux (Fig. 2). The number of crystals was chosen as a compromise between collection efficiency and price. All five crystals are located on Rowland circles of the same radius $R$ and reflect x-rays at the same Bragg angle $\theta$. The axis orientation in Fig. 1 corresponds to the middle crystal. The planes of the Rowland circles cross each other at the line $S D$. All the crystal surface normals $C_{i} O$ cross at point $O(i=1-5$ denotes the number of the crystal, ordered in $z$ ). Scanning the energy of the emitted radiation is performed by changing the Bragg angle of the crystal, i.e., by changing the positions of the detector and crystals in such a way that the Rowland geometry is retained and line $S C_{3}$ is always parallel to the $x$ axis. The position of the sample is not changed during the energy scan.

The position of the crystal center $C_{i}$ relative to the sample and orientation of the crystal are

$$
\begin{gathered}
x_{i}=2 R \sin (\theta) \cos ^{2}(\theta)+x p_{i} \sin (\theta), \\
y_{i}=2 R \cos (\theta) \sin ^{2}(\theta)-x p_{i} \cos (\theta), \\
z_{i}=(i-3) \mathrm{d} z,
\end{gathered}
$$

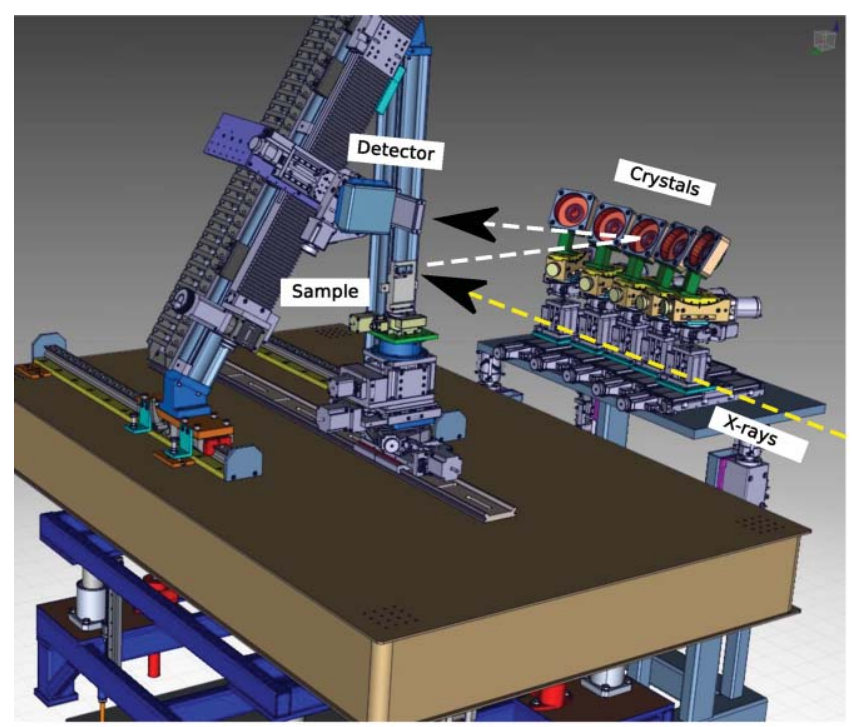

FIG. 2. (Color online) Scheme of the spectrometer as installed at the SuperXAS beamline.

$$
\begin{gathered}
\varphi_{i}=\pi-\arctan \left(z_{i} /\left(x p_{i} \sin (\theta)\right)\right), \\
\beta_{i}=\arctan \left(\frac{\sqrt{z_{i}^{2}+x p_{i}^{2} \sin ^{2}(\theta)}}{x p_{i} \cos (\theta)}\right), \\
x p_{i}=\sqrt{(2 R)^{2} \sin ^{4}(\theta)-z_{i}^{2}}, \\
L=2 R \sin (\theta)
\end{gathered}
$$

where $\varphi$ is the azimuthal angle of the crystal surface normal $\overrightarrow{C O}$ (in the plane $x z ; \varphi=0$ when $\overrightarrow{C O}$ is parallel to $x), \beta$ is its polar angle (between $\overrightarrow{C O}$ and $z$ ), $\mathrm{d} z$ is the distance between the crystals along $z$, and $L$ is the distance between the sample and crystal center, which is equal to the distance between the crystal and detector centers. Figure 3 shows a scheme of the crystal manipulator. The positions of the crystals along the $x$ axis are scanned with a horizontal $\left(X_{i}\right)$ translation stage, those along the $y$ axis with a vertical $\left(Y_{i}\right)$ translation stage mounted on top of the $X_{i}$ stage. The orientation of the crystal is adjusted with a horizontal $\left(\varphi_{i}\right)$ rotation stage mounted on top of the $Y_{i}$ stage and a vertical goniometer $\left(\beta_{i}\right)$ stage mounted on top of the $\varphi_{i}$ stage. The step size and repeatability of the moving stages do not exceed $5 \mu \mathrm{m}$, $0.1 \mu \mathrm{m}, 0.003^{\circ}$, and $0.001^{\circ}$ for $X, Y, \varphi$, and $\beta$, respectively. The $X, Y$, and $\beta$ stages are encoded and operated in the closeloop mode. The wobble of the stages is less than $0.004^{\circ}$.

The position and orientation of the detector relative to the sample are

$$
\begin{gathered}
x_{\mathrm{d}}=4 R \sin (\theta) \cos ^{2}(\theta), \\
y_{\mathrm{d}}=4 R \cos (\theta) \sin ^{2}(\theta), \\
z_{\mathrm{d}}=0, \\
\varphi_{\mathrm{d}}=\pi,
\end{gathered}
$$

$$
\beta_{\mathrm{d}}=(3 / 2) \pi-2 \theta \text {. }
$$

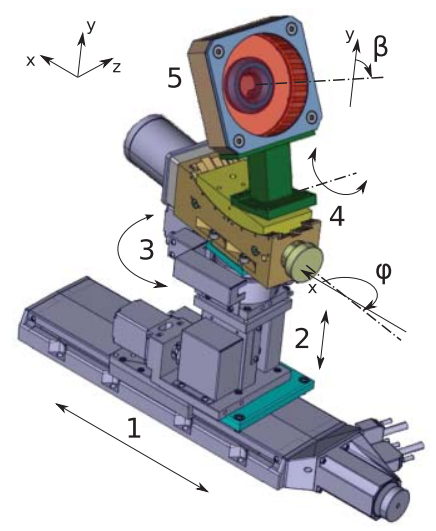

FIG. 3. (Color online) Crystal manipulator. 1: $X_{i}$ translation stage, 2: $Y_{i}$ translation stage, 3: $\varphi_{i}$ rotation stage, 4: $\beta_{i}$ goniometer stage, and 5: crystal holder. 
The position of the detector is scanned with two translation stages $X_{\mathrm{d}}^{\prime}$ and $Y_{\mathrm{d}}^{\prime}$, which are perpendicular to each other, and with the $X_{\mathrm{d}}^{\prime}$ axis tilted $60^{\circ}$ relative to the horizontal plane. The $Y_{\mathrm{d}}^{\prime}$ stage is mounted on the $X_{\mathrm{d}}^{\prime}$ stage. The orientation of the detector is adjusted by a $\left(\beta_{\mathrm{d}}\right)$ rotation stage mounted on the $Y_{\mathrm{d}}^{\prime}$ stage.

The radius $R(50 \mathrm{~cm})$ of the Rowland circle and diameter of the crystal $(10 \mathrm{~cm})$ are a compromise between the resolution of the spectrometer and its collection efficiency. The radius corresponds to a radius of curvature of the crystal of $100 \mathrm{~cm}$. The $\mathrm{d} z$ parameter was set at $130 \mathrm{~mm}$.

Five $\operatorname{Si}(111)$ crystals were produced by attaching opticalquality polished $\mathrm{Si}(111)$ wafers $(0.5-\mathrm{mm}$ thick) to opticalquality concave glass substrates. The production process described below is also applicable to other wafer materials. The production was performed in a class 10 cleanroom to avoid distortion of the crystals by contamination with particles on the wafer or glass surfaces. The wafer was spin-coated with epoxy glue and pressed onto the substrate with a "Teflon" (polytetrafluoroethylene) membrane by pressurized air. The volume between the wafer and the glass substrate was evacuated before and during pressurization to eliminate all the air. The crystal was heated to $120{ }^{\circ} \mathrm{C}$ and kept under pressure for several hours. The thickness of the glue was controlled by Newton interferometric rings, observed from the back of the substrate. The procedure resulted in a distance between the wafer and the substrate surfaces of less than $300 \mathrm{~nm}$ on more than $95 \%$ of the contact surface. Furthermore, several anodic-bonded crystals of different surface orientation were obtained from commercial suppliers. The home-made crystals provided a $0.3 \mathrm{eV}$ larger energy bandwidth and $10 \%$ lower collection efficiency of the spectrometer at $\mathrm{Cu} \mathrm{K} \alpha_{1}$ as compared to a commercial $\mathrm{Si}(111)$ crystal. This is due to the considerable waviness of the surface of the wafer, $\pm 0.2 \mathrm{mrad}$ for the wafers used and less than $\pm 0.04 \mathrm{mrad}$ for the wafers of commercial crystals, as measured with a laser long-trace profiler. The resolution and calibration given below were measured with a commercial crystal, and all the measurements involving five crystals were performed with the home-made $\mathrm{Si}(111)$ crystals.

The spectrometer was tested with two types of $\mathrm{x}$-ray detectors developed at the Paul Scherrer Institute: a twodimensional pixel detector PILATUS 100K (Ref. 18) and a one-dimensional strip detector MYTHEN II. ${ }^{19}$ Both are silicon detectors operated in photon-counting mode with energy discrimination. Each pixel or strip has its own amplifier, discriminator, and counting circuit. The maximal count rate of both detectors is above $1 \mathrm{MHz} /$ pixel. The dead time of the counting electronics is well below $1 \mu \mathrm{s}$, which corresponds to the linear range of the detector larger than $10^{5}$ count/s/pixel. The thickness of the silicon matrix of the detector modules tested is $0.3 \mathrm{~mm}$. Modules with a thicker silicon matrix are available from the SLS Detector Group or from Dectris Ltd. The pixel size of the PILATUS module is $0.172 \times 0.172 \mathrm{~mm}^{2}$, that of the MYTHEN module $0.05 \times 8 \mathrm{~mm}^{2}$. The matrix size is $487 \times 195$ pixels and 1280 strips, respectively. The design of the spectrometer enables the use of other types of detectors, including avalanche photodiodes.
The spectrometer consists of three units: a crystal table, a sample manipulator, and a detector manipulator. Each unit can be easily shifted from the working position to give access to another type of experiment at the beamline. The detectorand sample- manipulators are mounted on rails for long-range movement along the axis of the beam. The crystal table is equipped with air pads and can be easily moved by one person within the experimental hutch. The working position of the crystal table is fixed by spacers mounted on the experimental table.

The alignment of the spectrometer is achieved as follows. A pinhole aperture is placed at the sample position at $45^{\circ}$ to the beam and aligned so that the x-ray beam passes through the hole. The crystal manipulators are placed in the position, which corresponds to $\theta=90^{\circ}$ (Eqs. (1) and (2)). Metal cylinders with a mark at the center are used instead of the crystals. A construction laser-line level with a precision of $1.5 \mathrm{~mm} / 5$ $\mathrm{m}$ is used to adjust the crystal height. The height of the crystal table is adjusted so that the center of the middle crystal is at the same height as the pinhole. The tilt of the table is zero, as measured with two perpendicular linear bubble levels with an accuracy of $0.1 \mathrm{~mm} / \mathrm{m}$ mounted on the top of the table. The vertical position of each crystal is adjusted so that the crystal center is at the same height as the pinhole. A parallel laser-pointer beam is directed through the pinhole to the center of the middle crystal. The sample is aligned to the $x$ axis of the middle crystal by an iterative procedure: moving the crystal along the $x$ axis from limit to limit, monitoring the shift of the laser spot at the crystal, compensating the shift by changing the position of the sample along the beam, repeating the procedure until the shift is negligible. The crystals are mounted. The $\varphi$ and $\beta$ angles of the crystals are adjusted by back-reflection of the laser beam. The crystals and detector are put in the position, which corresponds to the desired $\theta$ (Eqs. (1) and (2)). The position of the detector is adjusted so that the laser beam, which is reflected by the middle crystal, is in the middle of the detector. The fine alignment is performed with $x$-rays that are elastically scattered from the sample. The positions of the crystals and the detector are adjusted to maximize the signal from the detector. The typical time to install and align the spectrometer is $8 \mathrm{~h}$.

\section{PERFORMANCE OF THE SPECTROMETER}

\section{A. Energy resolution}

The energy resolution (bandwidth) of a Johann-type spectrometer is influenced by the intrinsic resolution of the crystal, the quality of the crystal surface, the x-ray spot size on the sample, aberrations, and misalignment of the spectrometer. ${ }^{3,8,20}$

The intrinsic resolution of the crystal is the contribution to the energy bandwidth resulting from the properties of the crystalline material (excluding waviness of the surface). In our case, the major contribution to the intrinsic bandwidth is from stress in the lattice caused by the bending of the crystal wafer into a spherical shape. The dotted line in Fig. 4 shows the intrinsic bandwidth of a fourth diffraction-order reflection of a $\mathrm{Si}(111)$ wafer, $0.5-\mathrm{mm}$ thick, with a radius of curvature of 


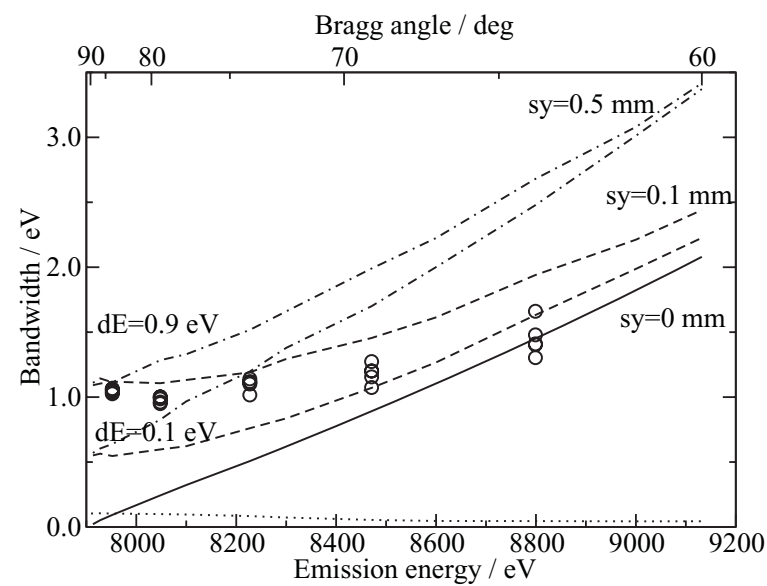

FIG. 4. Spectrometer resolution with a $\mathrm{Si}(111)$ crystal in the fourth diffraction order. Circles: measured bandwidth of elastically scattered radiation. Dotted line: intrinsic bandwidth of the crystal simulated with XOP. Solid line: contribution of aberrations (ray-tracing). Dashed and dashed/dotted lines: convolution of aberrations, finite vertical spot size ( $\mathrm{s} y=0.1$ and $0.5 \mathrm{~mm}$ ), and finite incident radiation bandwidth $(\mathrm{d} E=0.4$ and $0.9 \mathrm{eV})$ contributions.

$100 \mathrm{~cm}$, as simulated according to the multi-lamellar method and by using the XCRYSTAL_BENT module (Ref. 21) of the XOP package. ${ }^{22}$ The calculated energy broadening does not exceed $0.11 \mathrm{eV}$. The reported intrinsic broadening of similar bent crystals is in the range of $0.1-0.3 \mathrm{eV}^{8,20,23}$

An analysis of the influence of aberrations, source size, and misalignment of the spectrometer on its resolution was performed by several research groups. ${ }^{3,8,20}$ The best resolution can be achieved with a large radius $R$, with a small crystal diameter $d$, and close to back-scattering $\left(\theta=90^{\circ}\right)$. Figure 4 shows the theoretically calculated spectrometer resolution and experimentally measured bandwidth of elastically scattered beamline radiation. The measured bandwidth is a convolution of the incident energy bandwidth and that of the spectrometer. The solid line represents the contribution of the aberrations of the optical system, which increase with decreasing Bragg angle and vanish in the back-reflection geometry. To simulate experimental data, the finite source size (x-ray spot size at the sample) and the bandwidth of the incident radiation were assumed. The simulation with a beamline bandwidth of $0.9 \mathrm{eV}$ and spot size of $0.1 \mathrm{~mm}$, which are close to the estimated beamline parameters, provides the best fit of the experimental data. Based on that fit, the resolution of the spectrometer at $80^{\circ}$ is estimated to be below $0.5 \mathrm{eV}$, which corresponds to $\mathrm{d} E / E<6 \times 10^{-5}$.

Misalignment of the spectrometer elements will result in an error in the calibration of the spectrometer and broadening of the bandwidth. The major contribution to the energy broadening is from the radial shift $\mathrm{d} R$ of the sample (or respective shift of the crystal) from the Rowland circle. The projections of shifts of the sample (or respective shifts of the crystal) along the $x, y$, and $z$ axes on the Rowland circle radius are

$$
\mathrm{d} x_{R}=\left(\sin (\theta) \cos (2 \theta) \sqrt{1-g^{2}(\theta)}-\cos (\theta) \sin (2 \theta)\right) \mathrm{d} x,
$$

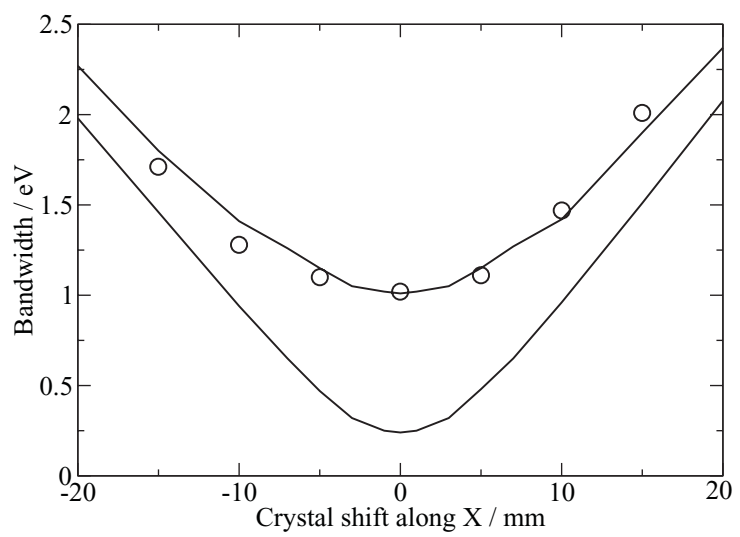

FIG. 5. Dependence of spectrometer bandwidth on misalignment along the $x$ axis. Circles: measured bandwidth of elastically scattered radiation. Solid line: ray-tracing simulation. Dashed line: convolution of the simulation with a finite bandwidth of the incident radiation $(0.9 \mathrm{eV})$.

$$
\mathrm{d} y_{R}=\left(-\cos (\theta) \cos (2 \theta) \sqrt{1-g^{2}(\theta)}-\sin (\theta) \sin (2 \theta)\right) \mathrm{d} y,
$$

$$
\begin{gathered}
\mathrm{d} z_{R}=(-g(\theta) \cos (2 \theta)) \mathrm{d} z, \\
g(\theta)=\frac{z_{i}}{2 R \sin (\theta) .}
\end{gathered}
$$

For the presented spectrometer design, $g|(\theta)|<0.1$. Therefore, the dependence of the energy broadening on the shift $\mathrm{d} x$ or $\mathrm{d} y$ will be nearly the same for all the crystals. Figure 5 shows the dependence of the width of an elastic peak measured with a $\mathrm{Si}(111)$ crystal in the fourth diffraction order at $E=8048 \mathrm{eV}$ on the position of the crystal along the $x$ axis. The dependence of the spectrometer bandwidth on crystal misplacement along the $y$ and $z$ axes is much weaker than that along the $x$ axis. Moreover, the alignment procedure ensures that misplacements $\mathrm{d} y$ and $\mathrm{d} z$ are less than $5 \mathrm{~mm}$. No significant broadening of the elastic peak was observed at $y$ shifts of up to $5 \mathrm{~mm}$. Misalignment of $\varphi$ by up to $1^{\circ}$ did not result in noticeable broadening either.

Another factor, which influences the overall bandwidth of the spectrometer is the spread of the energy calibration error of the crystals, which will be discussed below.

\section{B. Energy calibration}

The energy of the spectrometer is set by placing the elements of the spectrometer in the positions defined by Eqs. (1) and (2). Deviation of the actual positions from the nominal value causes an error in the energy. This error in position originates from imperfection of the elements and misalignment of the spectrometer. The total error, resulting from the imperfections in the elements, mounting errors, and alignment, was estimated to be $\mathrm{d} \theta<0.5 \mathrm{mrad}$ for on the full range of Bragg angles, which corresponds to an error of less than $2.6 \mathrm{eV}$ with a $\mathrm{Si}(111)$ crystal in the fourth diffraction order.

The calibration error was measured by checking the energy of the elastic peak at different incident energies (Fig. 6). The energy of the beamline was calibrated by the 


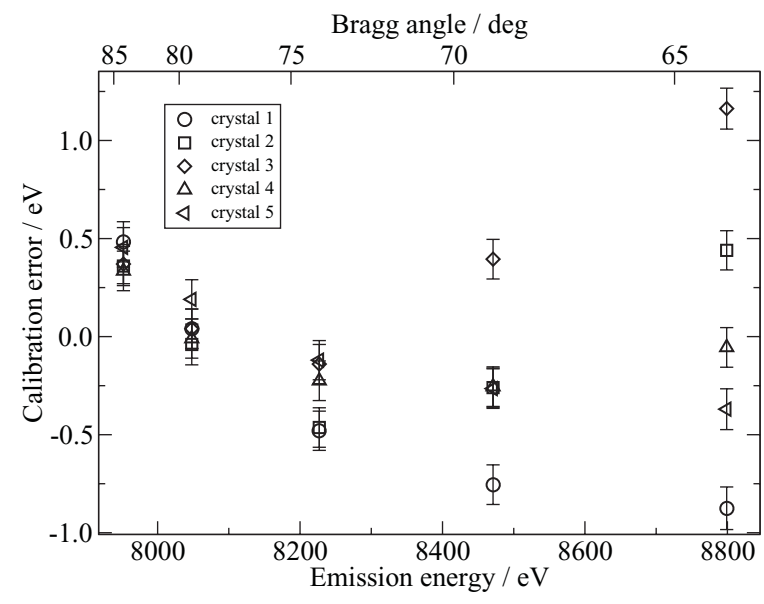

FIG. 6. Energy calibration error measured with elastically scattered x-ray radiation detected with a $\mathrm{Si}(111)$ crystal in the fourth diffraction order.

inflection point in the transmission-detected K-edge XAS of reference foils of $\mathrm{Co}, \mathrm{Ni}$, and $\mathrm{Cu}$ to give 7708.78(2), 8331.49(2), and 8980.48(2) $\mathrm{eV}$, respectively, ${ }^{24}$ with a precision of better than $0.1 \mathrm{eV}$. The spectrometer was calibrated at $8048 \mathrm{eV}\left(\theta=79.3^{\circ}\right)$. The absolute calibration error was less than $1.5 \mathrm{eV}$ for the full range of $\theta$, and the spread of the calibration errors of the crystals was less than $2.5 \mathrm{eV}$. Taking into account that the width of a typical scan in the vicinity of a fluorescence line does not exceed $50 \mathrm{eV}$, precision of the energy calibration of better than $0.2 \mathrm{eV}$ is typically achieved.

\section{Photon collection efficiency and signal-to-noise ratio}

The detector count rate $S$ depends on the following factors: emission photon flux $F_{\mathrm{e}}$, transmission by air and windows $T$, collection solid angle $\Omega$, integral reflectivity of the crystal $R I$, efficiency of the detector $D$. Assuming that the width of the emission line $\Delta E$ is much broader than the bandwidth of the spectrometer, the signal from the detector is estimated to be

$$
S=c \frac{F_{\mathrm{e}}}{\Delta E} \frac{\Omega}{4 \pi} T \cdot D \cdot R I \cdot E \cdot \cot \theta,
$$

where $c$ is a coefficient, which depends on the shape of the emission line. For the Gaussian-shaped emission line, $c=$ $4 \ln 2 / \sqrt{\pi} \approx 1.56$. The emitted flux $F_{\mathrm{e}}$ depends on the incident flux $F_{0}$,

$$
F_{\mathrm{e}}=F_{0} \cdot A_{\mathrm{elm}} \cdot Q Y \cdot S A,
$$

where $Q Y$ is the quantum yield, $S A$ is the self-absorption coefficient, and $A_{\text {elm }}$ is the absorption by the element in question, which depends on the stoichiometry of the sample and the absorption cross section of elements (for a metal foil $A_{\text {elm }}$ equals total absorption).

A good figure of merit for characterizing the efficiency of photon collection of the instrument would be $S \cdot \Delta E / F_{\mathrm{e}}$. Figure 7 displays experimental and theoretical values of collection efficiency. The theoretical values, shown as points, were calculated according to Eq. (4) for all $\mathrm{K} \alpha_{1}, \mathrm{~K} \beta_{1}, \mathrm{~L} \alpha_{1}$, and $\mathrm{L} \beta_{2}$ emission lines in that energy region. Integral reflec-

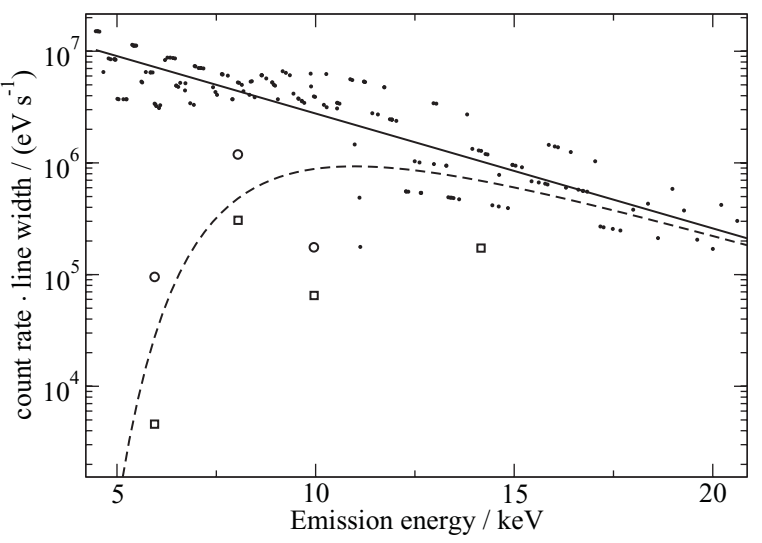

FIG. 7. Count rate at an emission flux of $10^{10}$ photon/s with five crystals. Points: calculated count rate in vacuum, solid line: linear fit of the calculated data, dashed line: the same, multiplied by x-ray transmission of air, squares: experiment in air, circles: experiment with a helium bag.

tivity $R I$ of appropriate $\left(85^{\circ}<\theta<75^{\circ}\right) \mathrm{Si}$ or Ge crystal cuts was calculated according to the multi-lamellar method using the XCRYSTAL_BENT module (Ref. 21) of XOP package. ${ }^{22}$ $R I$ depends on $\theta$, and on the cut orientation and the material of the crystal, all of which result in considerable scattering of the calculated points in the figure. The solid angle $\Omega$, covered by five crystals, is in the range $\left((3.1-3.6) \times 10^{-3} 4 \pi\right)$. The detector efficiency at low count rates can be approximated by the x-ray absorption of the silicon detecting element (0.3-mm thick), which is above $98 \%$ at emission energies below $8 \mathrm{keV}$ and decreases to $25 \%$ at $20 \mathrm{keV}^{25}$ The solid line in the figure represents a linear fit of the simulated data. The simulated collection efficiency decreases drastically with the emission energy. At $20 \mathrm{keV}$ it is two orders of magnitude lower than at $5 \mathrm{keV}$. The dashed line represents the collection efficiency, with $\mathrm{x}$-ray transmission by air $^{25}$ taken into account. Absorption by air is significant at energies below $12 \mathrm{keV}$. In order to decrease the absorption, a bag with kapton windows filled with helium is placed between the sample, the crystals, and the detector. The helium bag increases the count rate by factors of 4 and 20 at 8 and $6 \mathrm{keV}$, respectively. The emission energy range, for which the spectrometer is optimal in practical applications, was approximately 5 to $19 \mathrm{keV}$. Some spectra of reasonable quality were recorded at energies below $5 \mathrm{keV}$ and above $19 \mathrm{keV}$. Placing the spectrometer in a chamber filled with helium or under vacuum would extend that range to lower energies.

A typical count rate with a copper foil at $\mathrm{Cu} \mathrm{K} \alpha_{1}$ at an incident flux of $2 \times 10^{11}$ photon/s is $1.3 \times 10^{6} \mathrm{count} / \mathrm{s}$. The beamline flux was calculated from the current of a photodiode at the position of the sample. The calculation of the emitted flux was based on the measured absorption by the sample, published data on absorption cross sections, ${ }^{26,27}$ and quantum yield. ${ }^{28}$ The experimentally determined collection efficiency (squares and circles in Fig. 7) is significantly lower than the theoretical value. This disagreement may originate from imperfections in the crystals and uncertainty with regard to the parameters used in the calculations.

The sensitivity of the instrument with regard to low flux is limited by the background signal. The background origi- 


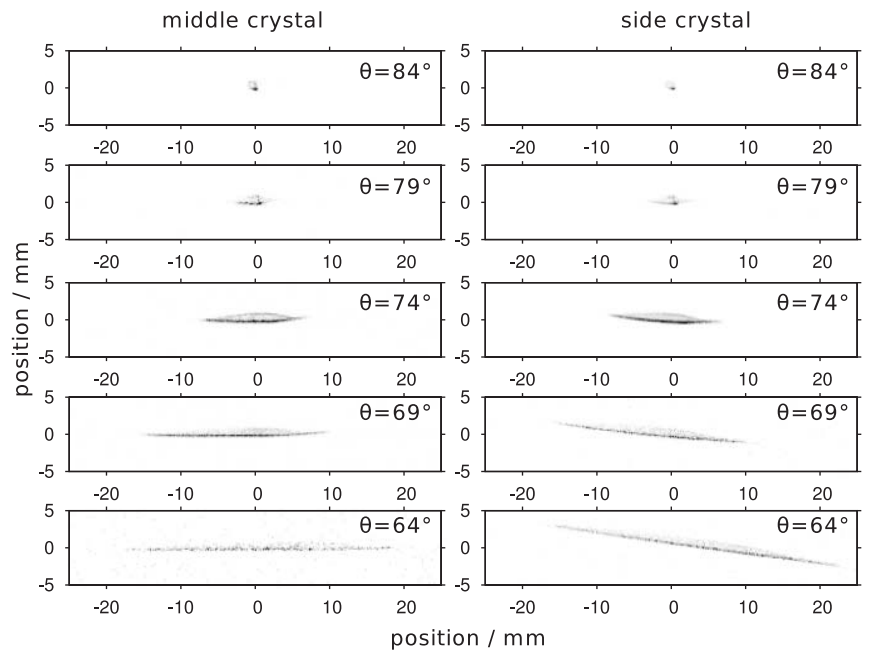

FIG. 8. Gray-scale map of x-ray intensity at the detector with a crystal in the middle position (left) and one at the side (right).

nates from the single scattering of emitted $\mathrm{x}$-rays and multiple scattering of the incident beam by the air and surfaces. The former represents the major contribution to the background at a high-emission flux, while the latter dominates at low emission flux. To optimize the signal-to-background ratio, the area of the detector should correspond to the size of the x-ray spot at the detector. Figure 8 gives the intensity map of the reflection spot of one crystal at the detector. The images were taken with elastically scattered $x$-ray radiation of the beamline. The spot size increases drastically with decreasing Bragg angle and is caused by astigmatism in the optical system, i.e., different focal distances of the system in the plane of the Rowland circle and in the plane perpendicular to it. The spot from the side crystal is tilted relative to that of the middle crystal, which corresponds to the tilt of the Rowland circle planes. A pixel (PILATUS) detector enables the fast and easy selection of the region of interest; thus, operation is always at an optimal signal-to-background ratio. With a strip (MYTHEN) detector one can set a region of interest vertically, while the horizontal strip size is always equal to $8 \mathrm{~mm}$, which is optimal for the Bragg angle range of $75^{\circ}-80^{\circ}$. The sensitivity of the spectrometer was tested by measuring the signal-tobackground ratio at the $\mathrm{Cu} \mathrm{K} \alpha_{1}$ line of copper oxide diluted with cellulose or cellulose and cerium oxide. The absorption of x-rays by copper $A_{\mathrm{Cu}}$ (see Eq. (5)) was in the range of 0.010.8 . The data were extrapolated to lower absorption, and a detection limit of 0.004 was found when defined as absorption by copper for a signal-to-background ratio of 10 , corresponds approximately to a concentration of $0.4 \mathrm{wt} \%$ copper. The sensitivity can be improved further by a factor of 5 by blocking the scattered radiation with an aperture in front of the sample.

\section{Examples of experimental data}

Figure 9 shows the $\mathrm{W} L \beta_{2}$ and $\mathrm{W}$ VB- $\mathrm{L}_{3}$ emission spectra of $\mathrm{Na}_{2} \mathrm{WO}_{4} \cdot 2\left(\mathrm{H}_{2} \mathrm{O}\right)$ recorded with five $\mathrm{Si}(111)$ crystals in the fifth diffraction order and by means of a helium bag. The excitation energy was $0.1 \mathrm{keV}$ above the $\mathrm{W} \mathrm{L}_{3}$ edge. The measured incident flux was $4 \times 10^{11}$ photon/s, and the collec-

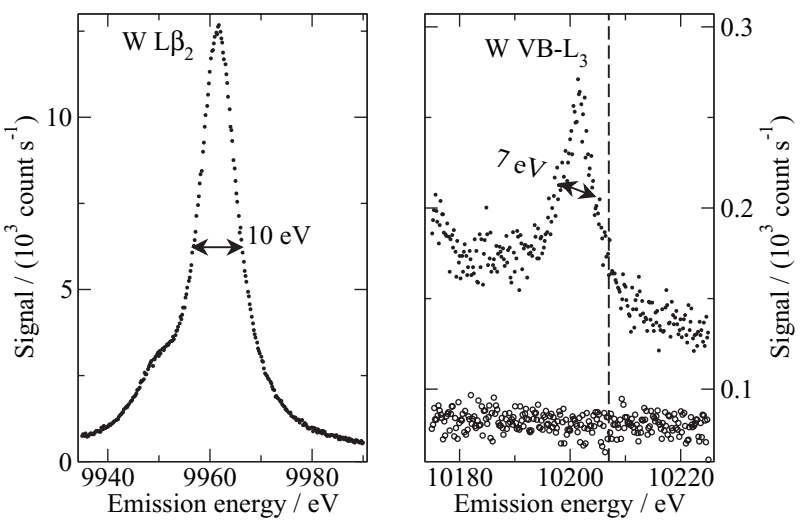

FIG. 9. W L $\beta_{2}$ and W VB- $\mathrm{L}_{3}$ emission spectra of $\mathrm{Na}_{2} \mathrm{WO}_{4} \cdot 2\left(\mathrm{H}_{2} \mathrm{O}\right)$ detected with five $\mathrm{Si}(111)$ crystals in the fifth diffraction order. The vertical dashed line indicates the energy of the $\mathrm{W} \mathrm{L}_{3}$ edge. The background signal is represented by open circles.

tion efficiency of the spectrometer at this energy was close to its optimum. The data were collected for 1 and $3 \mathrm{~s}$ per point in the $\mathrm{W} \mathrm{L} \beta_{2}$ and $\mathrm{W} V \mathrm{VB}-\mathrm{L}_{3}$ regions, respectively. The background signal (open circles) was collected at a random region on the detector several centimeters away from the region of interest, but covering the same area. The background signal does not seem to depend significantly on the emission energy in the W VB- $\mathrm{L}_{3}$ region, which indicates that the steep inclination of the emission spectrum in that region originates from the tail of the $\mathrm{W} \mathrm{L} \beta_{2}$ line.

Figure 10 shows an example of PFY XAS. The Ru Kedge of $\mathrm{RuO}_{2}$ was detected in transmission mode (solid line) and in PFY mode (dots) at the maximum of the $\mathrm{Ru} \mathrm{K} \alpha_{1}$ line. The fluorescence signal was averaged over $10 \mathrm{~s}$. The collection efficiency of the spectrometer at this energy is quite low (Fig. 7) because of the low crystal reflectivity and efficiency of the detector. PFY detection at such high energies is rare. Despite the low signal, the shape of the recorded spectrum is clearly distinguishable, and the features in the edge region of PFY XAS are sharper than those in the transmission-detected XAS spectra.

RIXS spectra (not shown here) are recorded by scanning the incident and emission energies. The intensity and

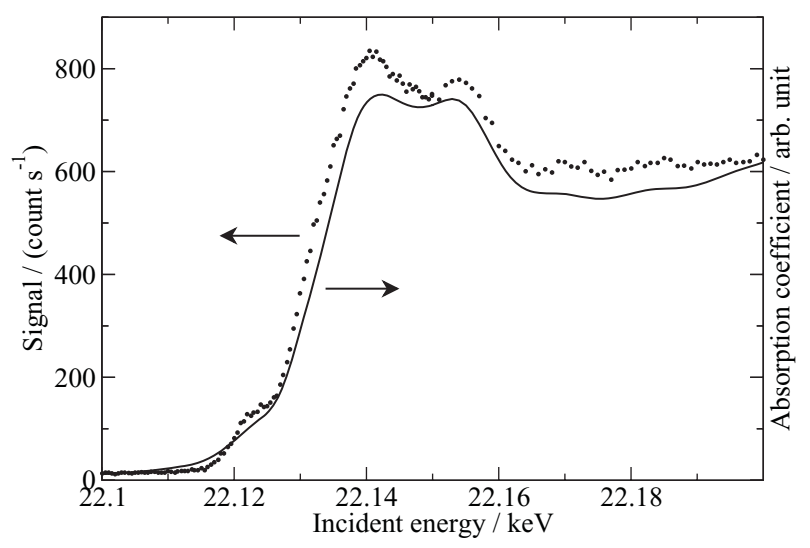

FIG. 10. Ru K-edge XAS of $\mathrm{RuO}_{2}$. Solid line: transmission-detected XAS, points: PFY XAS detected in the maximum of $\mathrm{Ru} \mathrm{K} \alpha_{1}$ with five $\operatorname{Si}(111)$ crystals in the ninth diffraction order. 


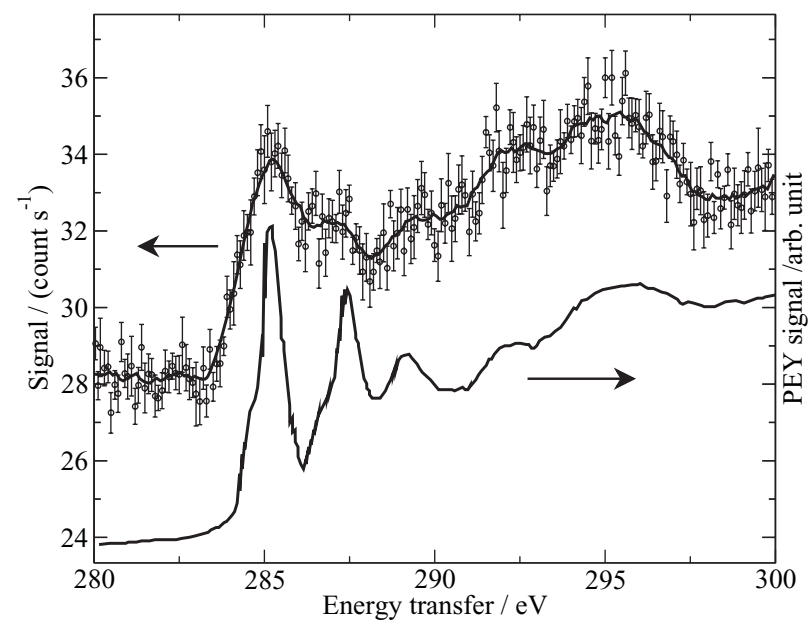

FIG. 11. Circles: C $1 s \mathrm{x}$-ray Raman spectrum of kapton. Error bars represent statistical error from 10 scans. Upper curve: smoothed data. Lower curve: C $1 s$ NEXAFS spectrum of kapton from Weiss $e t$ al. ${ }^{29}$

resolution in the RIXS spectra correspond to the XES and PFY XAS spectra. It typically takes $1 \mathrm{~h}$ to record a $\mathrm{Cu} 1 s 2 p$ RIXS plane of a copper foil.

It is also possible to detect non-resonant (Raman) x-ray scattering. Figure 11 shows a C $1 s$ XRS spectrum of a kapton foil, $100-\mu \mathrm{m}$ thick. The spectrum was recorded at an emission energy of $7950 \mathrm{eV}$ (Bragg angle of $84^{\circ}$ ). The incident flux was $4 \times 10^{11}$ photon/s. The data were collected for $8 \mathrm{~h}$. The spectrum is compared with a published C $1 s$ NEXAFS spectrum. ${ }^{29}$ All the main features in the NEXAFS are visible in the $x$-ray Raman spectrum, although with a lower energy resolution.

\section{CONCLUSION}

The constructed emission spectrometer enables recording XES, PFY XAS, RIXS, and XRS spectra with a subemission linewidth resolution in the energy range of $5-19 \mathrm{keV}$ or broader. A typical calibration error for an emission-line scan is below $0.2 \mathrm{eV}$. Using a pixel detector allows easy and fast optimization of the signal-to-background ratio. The concentration detection limit at $\mathrm{Cu} \mathrm{K} \alpha_{1}$ is below $0.4 \mathrm{wt} \%$.

\section{ACKNOWLEDGMENTS}

We thank Erich de Boni (PSI) for assistance with production of the crystals. Financial support was provided by the Swiss National Science Foundation (Grant No. 200021119881).
${ }^{1}$ P. Glatzel, F. M. F. de Groot, and U. Bergmann, Synchrotron Radiat. News 22, 12 (2009).

${ }^{2}$ M. O. Krause and J. H. Oliver, J. Phys. Chem. Ref. Data 8, 329 (1979).

${ }^{3}$ H. Johann, Z. Phys. 69, 185 (1931).

${ }^{4}$ L. V. von Hamos, J. Sci. Instrum. 15, 87 (1938).

${ }^{5}$ T. Johansson, Z. Phs. A: Hadrons Nucl. 82, 507 (1933).

${ }^{6}$ V. Stojanoff, K. Hamalainen, D. Siddons, J. Hastings, L. Berman, S. Cramer, and G. Smith, Rev. Sci. Instrum. 63, 1125 (1992).

${ }^{7}$ W. Schulke, A. Kaprolat, T. Fischer, K. Hoppner, and F. Wohlert, Rev. Sci. Instrum. 66, 2446 (1995).

${ }^{8}$ U. Bergmann and S. Cramer, Proc. SPIE 3448, 198 (1998).

${ }^{9}$ U. Bergmann, P. Glatzel, and S. P. Cramer, Microchem. J. 71, 221 (2002).

${ }^{10}$ H. Hayashi, M. Kawata, R. Takeda, Y. Udagawa, Y. Watanabe, T. Takano, S. Nanao, and N. Kawamura, J. Electron Spectrosc. Relat. Phenom. 136, 191 (2004).

${ }^{11}$ G. Tirao, G. Stutz, and C. Cusatis, J. Synchrotron Radiat. 11, 335 (2004).

${ }^{12}$ P. Glatzel and U. Bergmann, Coord. Chem. Rev. 249, 65 (2005).

${ }^{13}$ B. Dickinson, G. T. Seidler, Z. W. Webb, J. A. Bradley, K. P. Nagle, S. M. Heald, R. A. Gordon, and I. M. Chou, Rev. Sci. Instrum. 79, 123112 (2008).

${ }^{14}$ E. Kleimenov, A. Bergamaschi, J. van Bokhoven, M. Janousch, B. Schmitt, and M. Nachtegaal, J. Phys.: Conf. Ser. 190, 012035 (2009).

${ }^{15}$ L. Journel, L. E. Khoury, T. Marin, R. Guillemin, S. Carniato, A. Avila, R. Delaunay, C. F. Hague, and M. Simon, Rev. Sci. Instrum. 80, 093105 (2009).

${ }^{16}$ J.-L. Hazemann, O. Proux, V. Nassif, H. Palancher, E. Lahera, C. Da Silva, A. Braillard, D. Testemale, M.-A. Diot, I. Alliot, W. Del Net, A. Manceau, F. Gélébart, M. Morand, Q. Dermigny, and A. Shukla, J. Synchrotron Radiat. 16, 283 (2009).

${ }^{17}$ R. Verbeni, T. Pylkkänen, S. Huotari, L. Simonelli, G. Vankó, K. Martel, C. Henriquet, and G. Monaco, J. Synchrotron Radiat. 16, 469 (2009).

${ }^{18}$ P. Kraft, A. Bergamaschi, C. Broennimann, R. Dinapoli, E. F. Eikenberry, B. Henrich, I. Johnson, A. Mozzanica, C. M. Schlepütz, P. R. Willmott, and B. Schmitt, J. Synchrotron Radiat. 16, 368 (2009).

${ }^{19}$ A. Bergamaschi, A. Cervellino, R. Dinapoli, F. Gozzo, B. Henrich, I. Johnson, P. Kraft, A. Mozzanica, B. Schmitt, and X. Shi, J. Synchrotron Radiat. 17, 653 (2010).

${ }^{20}$ E. Collart, A. Shukla, F. Gelebart, M. Morand, C. Malgrange, N. Bardou, A. Madouri, and J. Pelouard, J. Synchrotron Radiat. 12, 473 (2005).

${ }^{21}$ M. S. del Rio, C. Ferrero, and V. Mocella, Proc. SPIE 3151, 312 (1997).

${ }^{22}$ M. Sanchez del Rio and R. J. Dejus, Proc. SPIE 3152, 148 (1997).

${ }^{23}$ K. Hämäläinen, D. P. Siddons, J. B. Hastings, and L. E. Berman, Phys. Rev. Lett. 67, 2850 (1991).

${ }^{24}$ S. Kraft, J. Stumpel, P. Becker, and U. Kuetgens, Rev. Sci. Instrum. 67, 681 (1996).

${ }^{25}$ B. L. Henke, E. M. Gullikson, and J. C. Davis, At. Data Nucl. Data Tables 54, 181 (1993).

${ }^{26}$ B. Ravel and M. Newville, J. Synchrotron Radiat. 12, 537 (2005).

${ }^{27}$ W. T. Elam, B. D. Ravel, and J. R. Sieber, Radiat. Phys. Chem. 63, 121 (2002).

${ }^{28}$ M. O. Krause, J. Phys. Chem. Ref. Data 8, 307 (1979).

${ }^{29}$ K. Weiss, C. Woll, E. Bohm, B. Fiebranz, G. Forstmann, B. Peng, V. Scheumann, and D. Johannsmann, Macromolecules 31, 1930 (1998). 